Pneumologe $2020 \cdot 17: 105-112$

https://doi.org/10.1007/s10405-019-00291-8

Online publiziert: 10. Dezember 2019

(c) Springer Medizin Verlag $\mathrm{GmbH}$, ein Teil von

Springer Nature 2019

\section{Redaktion}

G. Rohde, Frankfurt

\author{
Ruth Hörster ${ }^{1}$ Jan Rupp ${ }^{2,3}$ \\ ${ }^{1}$ Med. Klinik III/Pulmologie, Universitätsklinikum Schleswig-Holstein/Campus Lübeck, Lübeck, \\ Deutschland \\ ${ }^{2}$ Klinik für Infektiologie und Mikrobiologie, Universitätsklinikum Schleswig-Holstein/Campus Lübeck, \\ Lübeck, Deutschland \\ ${ }^{3}$ Deutsches Zentrum für Infektionsforschung, Hamburg-Lübeck-Borstel-Riems, Universität zu Lübeck, \\ Lübeck, Deutschland
}

\title{
Das neue Verständnis pulmonaler Infektionen
}

Immunsuppression, die sowohl ambulant als auch im Krankenhaus erworben sein können und die bislang in keiner der vorhandenen Pneumonie-Leitlinien umfassend abgehandelt sind.

In aktuellen Daten wird in Deutschland von über 660.000 Patienten mit CAP pro Jahr ausgegangen, wovon knapp die Hälfte der Patienten einer stationären Versorgung bedürfen. Die Sterblichkeit steigt drastisch bei älteren Patienten und vorliegenden Komorbiditäten. Insbesondere für chronische Herzinsuffizienz, Diabetes mellitus und Demenzerkrankungen konnte in verschiedenen Arbeiten eine erhöhte 1-Jahres-Mortalität gezeigt werden [15, 33]. Das Spektrum der bakteriellen Infektionserreger einer CAP hat sich in den vergangenen Jahren nicht grundlegend geändert. Die häufigsten Erreger sind weiterhin Streptococcus pneumoniae, Haemophilus influenzae, Staphylococcus aureus und Mycoplasma pneumoniae und bei älteren Patienten vermehrt Enterobacteriaceae. Entgegen des anfänglichen Trends nach Einführung der Impfung mit dem 13valenten Pneumokokken-Konjugatimpfstoff (PCV13) zeigen neuere Daten aus Großbritannien in den letzten Jahren eine erneute Zunahme an Pneumokokkenpneumonien. Hierbei fanden sich vermehrte Pneumokokken der nichtvakzinierten Serotypen, aber auch des Serotyps 3, der in dem Impfstoff PCV13 enthalten ist [22].

Der Anteil an gramnegativen Bakterien, wie E. coli, Klebsiella pneumoniae und Pseudomonaden sowie multiresistenter Erreger ist weiterhin gering und betrifft zumeist Patienten mit definierten Risikofaktoren (chronische obstruktive Lungenerkrankung [COPD], Bronchiektasen, PEG-Sonde [perkutane endoskopische Gastrostomie], u.a.; [5]). Eine vorbekannte Kolonisation mit Methicillin-resistentem Staphylococcus aureus (MRSA) im Respirationstrakt erhöht zwar das Risiko für eine Pneumonie durch MRSA, allerdings ist der positive Vorhersagewert nicht ausreichend hoch, als dass eine Leitlinienempfehlung für eine initial MRSA-wirksame Therapie bei diesen Patienten ausgesprochen wurde [5]. Ähnlich verhält es sich für eine der größten CAP-Populationen, den Patienten aus Senioren- und Pflegeheimen (früher „nursing home acquired pneumonia“, NHAP), die generell ein erhöhtes Risiko für die Besiedlung mit multiresistenten Erregern (MRE) aufweisen. Aufgrund vermehrter therapieassoziierter Nebenwirkungen hat es sich jedoch nicht bewährt, diese Patienten grundsätzlich mit einer MREwirksamen Therapie zu behandeln [5].

Umso wichtiger ist es, Patienten mit entsprechenden Risikofaktoren und Komorbiditäten im klinischen Alltag frühzeitig zu identifizieren, um den Besonderheiten dieser Personengruppe besser Rechnung tragen zu können.

Die Therapiedauer der Pneumonie richtet sich insbesondere nach den klinischen Stabilitätskriterien. Bei leichter bis mittelschwerer Pneumonie ist die Dauer der antimikrobiellen Therapie für 5-7 Tage empfohlen. Kürzere Therapien sind bei sehr frühzeitiger klinischer Stabilisierung möglich und können das Pneumonien unterschieden. Davon abgegrenzt werden Pneumonien unter 
Auftreten therapieassoziierter Nebenwirkungen, besonders bei älteren Patienten, verhindern. Stabilitätskriterien (wie Normalisierung der Herzfrequenz und des Blutdrucks, Atemfrequenz $<24 /$ min, Normothermie, Normalisierung des kognitiven Zustands auf Vorniveau und die Besserung der Hypoxämie) sollten mindestens 2 Tage vor Beendigung der antibiotischen Therapie erreicht werden [5]. Bei der schweren Pneumonie wird die antibiotische Therapie in der Regel ebenfalls nicht länger als sieben Tage durchgeführt. Auch hier ist die klinische Stabilisierung essenziell für die Dauer und Beendigung der Therapie [5].

\section{》) Eine klinische Stabilisierung ist essenziell für die Dauer und Beendigung der antibiotischen Therapie}

Von hoher klinischer und ökonomischer Relevanz ist die nosokomiale Pneumonie. Nach den Daten der aktuellen nationalen Prävalenzstudie zu nosokomialen Infektionen und Antibiotikaanwendung sind $24 \%$ aller im Krankenhaus erworbener Infektionen Infektionen der unteren Atemwege [20].

Häufige Erreger der im Krankenhaus erworbenen Pneumonie („hospital acquired pneumonia“, HAP) sind aerobe und fakultativ anaerobe gramnegative Stäbchenbakterien aus der Gruppe der Enterobacteriaceae (E. coli, K. pneumoniae und Enterobacter sp.) sowie Pseudomonas aeruginosa, Haemophilus influenzae, Acinetobacter baumannii und Stenotrophomonas maltophilia. Bei den grampositiven Erregern der nosokomialen Pneumonien finden sich häufig Staphylococcus aureus und Streptococcus pneumoniae.

Aufgrund des höheren Anteils an MRE als Ursache der nosokomialen Pneumonie muss bei entsprechendem Krankheitsverdacht unmittelbar das individuelle Risiko des Pateinten überprüft werden, um zeitnah eine entsprechende, kalkulierte und ggf. MRE-wirksame antibiotische Therapie einleiten zu können. Dabei ist zu berücksichtigen und als erhöhtes Risiko anzunehmen, ob und welche antibiotischen Vortherapien der Patient in den letzten 90 Tagen erhalten hat oder ob z.B. eine Kolonisation mit $P$. aeruginosa, multiresistenten gramnegativen Erregerkeimen (MRGN) oder MRSA vorliegt. Auch hier gilt es, zugrundeliegende strukturelle Lungenerkrankungen frühzeitig zu erkennen, um das Risiko einer initial nichtwirksamen Therapie besser abschätzen zu können. Ein weiterer relevanter Risikofaktor für Infektionen mit MRE ist die Dauer des stationären Aufenthalts vor dem Auftreten der Pneumonie. So werden Patienten mit einer HAP, die innerhalb der ersten 5 Tage nach Krankenhausaufnahme („early-onset“ HAP) erworben wird, von den Patienten, die nach dem 5. Aufenthaltstag im Krankenhaus („late-onset“ HAP) eine Pneumonie entwickeln, unterschieden. Klassische HAP-Erreger finden sich gehäuft bei Patienten mit längerem Krankenhausaufenthalt, während bei Patienten ohne Risikofaktoren für MRE und „early-onset“-HAP, Erreger wie bei der CAP im Vordergrund stehen [2].

Besondere Aufmerksamkeit ist bei Reiserückkehrern geboten, die eine medizinische Versorgung in Süd- und Osteuropa, Asien, Afrika oder im Nahen Osten erhalten haben, da diese Patienten ein besonders hohes MRE-Risiko haben.

\section{\) Patientenspezifische Risikofaktoren sind essenziell für die Therapiefindung}

Daraus erschließt sich, dass die Einschätzung und Evaluierung der Risikofaktoren des Patienten essenziell für die Therapiefindung sind. $\mathrm{Zu}$ den individuellen Risiken sowohl jüngerer, als auch älterer Patienten gehört die medikamentös induzierte Immunsuppression. Seit mehr als zehn Jahren ist ein kontinuierlicher Verbrauchsanstieg von Immunsuppressiva $\mathrm{zu}$ verzeichnen. Im Jahr 2017 erreichte der Verbrauch einen Wert von 187,2 Mio. definierten Tagesdosierungen (DDD), was einem Zuwachs von 11,3 Mio. DDD bedeutet. Die häufigsten verordneten Immunsuppressiva (Tumortherapien ausgenommen) waren Methotrexat, Azathioprin, Adalimumab,
Leflunomid, Infliximab, MycophenolatMofetil (MMF) und Tacrolimus [11].

Aufgrund steigender Zahlen immunsupprimierter Patienten, besseren Überlebenschancen von therapierten Tumorpatienten und insgesamt zunehmender Alterung und Komorbidität der Bevölkerung, ist in der Diagnostik und der Therapie der pulmonalen Infektionskrankheiten an ein verschobenes Erregerspektrum zu denken.

\section{Molekulare Verfahren zur Erregerdiagnostik}

Mit dem Einzug molekularer Verfahren als Ergänzung zur bislang üblichen bakteriellen Erregerdiagnostik mittels kultureller Anzuchtverfahren, hat sich das Spektrum der für eine respiratorische Infektsymptomatik in Frage kommenden Pathogene deutlich erweitert. Was für epidemiologische Betrachtungen zunächst einmal hoffnungsvoll erscheint, da durch eine vermehrte Sensitivität vermeintlich eine größere Anzahl von Erregern entdeckt werden können, bereitet häufig Schwierigkeiten in der Interpretation. So können z.B. einzelne Viren nicht eindeutig als Krankheitserreger klassifiziert werden, da die Pathogenität bislang wenig untersucht wurde bzw. auch Vergleichskollektive von Patienten ohne entsprechende Symptomatik fehlen. In gleicherweise finden sich nun gehäuft Koinfektionen von mind. zwei Erregern, welche zuvor häufig auch mal übersehen werden konnten.

Der Nutzen molekularer Testverfahren für eine Optimierung der Therapie ist bislang nur wenig untersucht. Es fehlen prospektive Studien, die einen direkten Nutzen der zumeist in einem Multiplex-PCR-Ansatz durchgeführten Verfahren auf wichtige klinische Endpunkte wie Mortalität, stationäre Verweildauer oder auch den effizienteren Einsatz von Antibiotika zeigen. Eine Kombination von zeitnaher und sensitiver Erregerdiagnostik erscheint derzeit nur in Kombination mit neuen, prädiktiven Biomarkern eine Verbesserung aktueller Therapiealgorithmen zu erbringen [29].

In den aktuellen Leitlinien der Infectious Diseases Society of America/ American Thoracic Society (IDSA/ATS) 
finden sich somit auch nur eingeschränkte Empfehlungen zum Nutzen von molekularen Nachweisverfahren in der klinischen Routine bei Patienten mit CAP [19]. Insbesondere im Hinblick auf Zusatzinformationen bzgl. des Vorhandenseins einer virusassoziierten Infektion der Lunge ist bei Patienten mit einer Immunsuppression der Einsatz von molekularen Testverfahren zu rechtfertigen. Dabei gibt es eine gute Übereinstimmung in der Testung von Abstrichen des Nasopharynx (NP) und der Analyse aus bronchoalveolärer Lavage (BAL) zu Beginn der klinischen Symptomatik [16]. Bei klinischer Verschlechterung und dann sequentiell durchgeführter Bronchoskopie waren jedoch in der BAL innerhalb von 7 Tagen auch Erreger nachweisbar, die initial in NP nicht gefunden werden konnten. Es wird deshalb in weiteren Studien zu prüfen sein, ob eine Stufendiagnostik aus NP- und BAL-Testungen im klinischen Verlauf den erwünschten Effekt zeigt und welchen Nutzen sie im Hinblick auf direkte Therapieentscheidungen erbringt.

\section{Pulmonale Infektionen durch Viren}

Bei großen epidemiologischen Untersuchungen mit molekularen Testverfahren wurde bei CAP-Patienten eine Vielzahl unterschiedlichster Viren gefunden. Auch wenn dies im klinischen Alltag bislang nicht zur gängigen Praxis gehört, stellen sich zentrale Fragen zur Bedeutung der Virusnachweise im klinischen Kontext und wie virale Pneumonien von gemischten viral-bakteriellen Pneumonien und der bakteriellen Pneumonie zu unterscheiden sind. Es ist davon auszugehen, dass der zunehmende Nachweis viraler Erreger zunächst Ausdruck der verbesserten diagnostischen Möglichkeit ist. In mehreren Studien gelang bei bis zu $30 \%$ der Patienten mit CAP der Nachweis von Viren, darunter v. a. Influenza A/B, Rhinovirus, RSV (Respiratory Syncytial Virus), Coronaviren und humanes Metapneumovirus [27]. Wie allgemein bekannt, weisen viral-bakterielle Koinfektionen starke saisonale Unterschiede auf und treten auf der Nordhalbkugel mit einer Häufung im Winter und Frühling

Pneumologe 2020 - 17:105-112 https://doi.org/10.1007/s10405-019-00291-8

(c) Springer Medizin Verlag GmbH, ein Teil von Springer Nature 2019

\section{R. Hörster · J. Rupp}

\section{Das neue Verständnis pulmonaler Infektionen}

\section{Zusammenfassung}

Epidemiologische Daten zur Erregerverteilung stellen bei respiratorischen Infektionen noch immer die Grundlage für empirische Therapieempfehlungen dar. Durch rasante technologische Entwicklungen im Bereich der Multiplex-und Sequenzierverfahren erweitert sich zunehmend das Spektrum potenziell relevanter Erreger und stellt bisherige Dogmen zur Virulenz und Pathogenität einzelner Erreger auf die Probe. Dabei behalten klassische pathogene Erreger der Lunge ihre Bedeutung, werden jedoch zunehmend in einen Kontext gesetzt, der virale Koinfektionen und Veränderungen des lokalen Mikrobioms berücksichtigt. Neuere Daten legen nahe, dass es erst durch die Integration dieser Informationen möglich sein wird, saisonale Unterschiede in der Häufigkeit bestimmter Lungeninfektionen zu erklären und neue Ansätze zur Stratifizierung von Risikopatienten zu finden. Dies wird besonders offensichtlich bei immunsupprimierten Patienten, bei denen Infektionen mit Viren und fakultativ pathogenen Erregern wie nichttuberkulösen Mykobakterien (NTM) zu schweren Krankheitsverläufen mit hoher Morbidität und Letalität führen können. Auf der Basis grundlegender Kenntnisse zum Erregerspektrum von ambulant und nosokomial erworbenen Pneumonien werden neue Ansätze in der Erregerdiagnostik und der Analytik des Lungenmikrobioms diskutiert und hinsichtlich der aktuellen Anwendbarkeit im Alltag hinterfragt.

\section{Schlüsselwörter}

Respiratorische Infektionen - Erregerdiagnostik · Viren · Nichttuberkulöse Mykobakterien . Mikrobiom

\section{The new comprehension of pulmonary infections}

\section{Abstract}

Epidemiological data on the distribution of mostly bacterial pathogens are still the basis for empirical treatment recommendations on respiratory infections. Because of the dynamic technological developments in molecular multiplexing and sequencing procedures, the spectrum of potential pathogens is increased and challenges the current dogma of virulence and pathogenicity of certain pathogens. Classical pathogens of the lungs are thereby not questioned but are increasingly placed in a context that reflects co-infections with viruses and changes of the local microbiome in more depth. Recent data indicate that integration of this novel information is required for a better understanding of the seasonal differences in the frequency of particular lung infections and to find new approaches to risk stratification of patients.
This becomes most obvious in the subgroup of immunosuppressed patients who are at risk of severe courses of diseases with higher morbidity and mortality from infections with viruses and facultative pathogens, such as nontuberculous mycobacteria (NTM). Based on the fundamental knowledge on the spectrum of pathogens of communityacquired and nosocomial lung infections, novel approaches in pathogen diagnostics and lung microbiome analytics are discussed and the applicability with respect to the current clinical routine is questioned.

\section{Keywords}

Respiratory infections - Pathogen detection . Viruses - Nontuberculous mycobacteria . Microbiome und einem Peak im 1. Quartal des Jahres auf. Während dieser Zeit sind Koinfektionen sogar häufiger als jede Infektion für sich [13]. Häufungen von Infektionen mit S. pneumoniae während der kalten Jahreszeit sind zeitlich mit der saisonalen Influenza assoziiert. Sowohl für die Kolonisation mit S. pneumoniae, als auch für die invasive Pneumokokkeninfektion gelten saisonale Unterschiede. Auffällig ist, dass begleitende/vorangehende grippeähnliche Beschwerden (plötzlich einsetzendes Fieber $\geq 39^{\circ} \mathrm{C}$ mit Myalgien und respiratorischen Symptomen) als Ausdruck einer simultanen Virusinfektion mit einem deutlich erhöhten Risiko für eine invasive Pneumokokkeninfektion einhergehen [4]. 


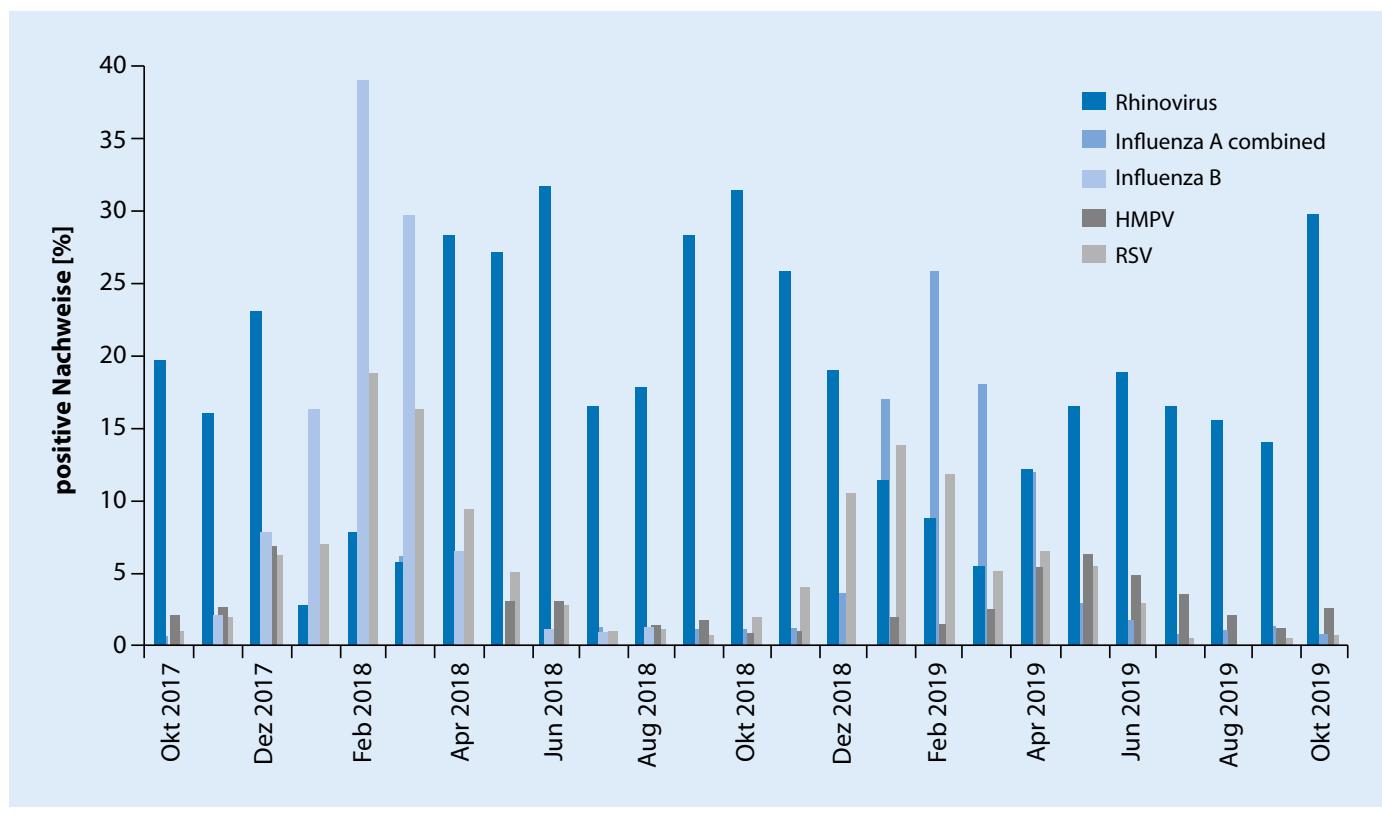

Abb. $1<$ Saisonale Verteilung von Influenza $A / B$, Rhinovirus, humanen Metapneumovirus (HMPV) und Respiratory Syncytial Virus (RSV) über den Zeitraum von Oktober 2017 bis Oktober 2019 aus RespVir, einem Zusammenschluss verschiedener Laboratorien und Institute aus Europa (Datenquelle: clinicalvirology.net/de)
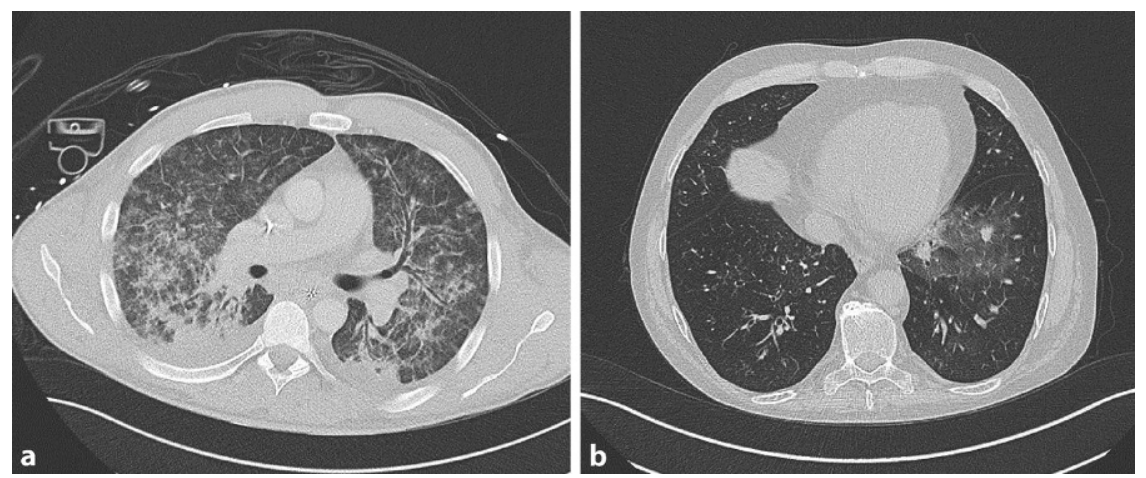

Abb. $2 \Delta$ a Patient mit schwerer bakteriell-viraler Koinfektion mit invasiver Beatmung. b Viruspneumonie mit moderatem Sauerstoffbedarf

Ob die Koinfektion mit Viren nur ein Begleiteffekt der verbesserten Diagnostik ist oder ob einzelne, bislang eher als Kolonisation gewertete Viren auch eine pathogene Rolle bei der CAP spielen, wurde bislang nur in wenigen Studien untersucht. In einer großen, multizentrischen Studie (EPIC) an Patienten, die mit CAP im Krankenhaus behandelt wurden, zeigte sich bei den Patienten $>65$ Jahre eine fast 10-mal so hohe Inzidenz von Rhinoviren als bei den jüngeren Patienten [36]. In einer weiteren Studie wurden Rhinoviren bei 21 von 192 Erwachsenen mit CAP (10,9\%), aber nur bei 2 von 238 asymptomatischen Erwachsenen (0,8\%) nachgewiesen [27].

Diese Ergebnisse legen nahe, dass die virale Koinfektion (in diesem Fall mit Rhinovirus) eine pathogene Rolle spie- len kann und mit der CAP assoziiert ist. Die epidemiologische Verteilung unterschiedlicher Viren ist über viele Jahre mittlerweile gut dokumentiert (• Abb. 1).

Die Differenzierung und die Erkenntnis einer viral-bakteriellen Koinfektion sind von klinischer Relevanz (• Abb. 2), da die viral-bakteriellen Pneumonien schwerwiegender verlaufen und zu längeren Krankenhausaufenthalten führen, als bakterielle Pneumonien allein [14]. In einer kleinen Studie aus Singapur war der Nachweis einer Koinfektion von Bakterien - insbesondere mit Influenzaviren ten mit schwerer CAP vergesellschaftet [24].

Ob sich aus einer schnelleren und umfangreicheren viralen Diagnostik auch eine Änderung von Antibiotikaregimen mit einer erhöhten Mortalität bei Patien- und möglicherweise eine Reduktion des Antibiotikaeinsatzes ergibt, und ob sich daran auch die Möglichkeit oder Notwendigkeit einer antiviralen Therapie schließt, müssen weitere Studien zeigen.

\section{》) Virale Koinfektionen sind mit CAP assoziiert}

Hilfreich könnte ein erweitertes diagnostisches Spektrum für Viren bei Krankenhauspatienten aus epidemiologischen und hygienischen Gründen sein. Je nach Erregernachweis können daraus ggf. Maßnahmen zur Infektionskontrolle resultieren, um eine potenzielle nosokomiale Übertragung zu verhindern [3]. Die frühe Kenntnis virulenter Erreger wäre sicherlich auch bei Patienten, die in Gemeinschaftseinrichtungen leben, vorteilhaft. So zeigte sich für RSV eine erhöhte Morbidität und Mortalität bei Patienten, die in Pflegeeinrichtungen leben, mit einer Übertragungsrate von $12 \%$ und Mortalitätsrate von bis zu $38 \%$ [6].

Besondere Aufmerksamkeit bedarf es bei Häufungen respiratorischer Infektionspatienten außerhalb der typischen saisonalen Verteilungen oder auch bei Auftreten von bislang wenig bekannten Begleitsymptomen.

Insbesondere bei Patienten oder Reiserückkehrern aus dem mittleren Osten 
mit respiratorischen Symptomen (geschätzte Inkubationszeit 12 Tage) oder mit Kontakt zu einem Patienten mit bestätigter oder wahrscheinlicher MERSCoV-Infektion (Middle East Respiratory Syndrome Coronavirus) sollte eine gezielte Diagnostik für MERS-CoV und Isolation durchgeführt werden. Immunsuppression scheint auch hierbei ein Risikofaktor für die Infektion zu sein [10]. Es handelt sich insgesamt zwar um eine seltene Infektionskrankheit, die aber bei unzureichender Diagnostik und fehlenden Schutzmaßnahmen zu Ausbruchsgeschehen mit hoher Letalität führen kann. Auch bei Häufungen unklarer Infektionskrankheiten mit besonderen klinischen Symptomen sollte die Diagnostik erweitert werden. Seit 2014 wurden in Europa und den USA mehrere Ausbrüche, vorwiegend bei Kindern dokumentiert, mit z. T. sehr schwer verlaufenden Lungeninfektionen bei Enterovirus-Infektionen. Klinisch auffällig war, dass diese häufig mit neurologischen Symptomen, wie einer schlaffen Paralyse, einhergingen [8].

Durch den Einsatz molekularer Testverfahren werden signifikant mehr virale Erreger in Abstrichen des Oropharynx (OP) oder NP gefunden. Jedoch scheint der positive Virusnachweis in der PCR kein guter Marker für die Vorhersage einer Pneumonie zu sein. Prendki et al. haben dazu bei Patienten über 65 Jahre, die mit V.a. eine Pneumonie stationär aufgenommen wurden, einen NPAbstrich und zur Sicherung der Diagnose eine „Low-dose“-Computertomographie (CT) durchgeführt. Zwar steigt die Sensitivität der insgesamt gefundenen Erreger gegenüber der konventionellen kulturellen Testung für Bakterien an, dies geht jedoch mit einem drastischen Verlust der Spezifität einher, da auch Patienten mit positivem Virusnachweis ohne Hinweis auf das Vorliegen einer Pneumonie in der CT gefunden werden [23].

In einer Studie von Saarela et al. wurde untersucht, ob durch das Vorliegen einer Multiplex-Virus-PCR von 16 verschiedenen Viren innerhalb einer Zeit von $24 \mathrm{~h}$ nach Eintreffen des symptomatischen Patienten in der Notaufnahme, die mittlere Verweildauer des Patienten oder der Antibiotikaverbrauch reduziert werden kann. Obwohl in 16-19\% der Patienten Viren im oberen Respirationstrakt gefunden wurden, hatte dies keinen Einfluss auf die beiden genannten Faktoren. Limitationen waren die insgesamt lange Therapiedauer von 10,4-11,3 Tagen in beiden Gruppen und fehlende konkrete Handlungsanweisungen, wie im Fall eines Virusnachweises zu verfahren ist [26]. In einer anderen Arbeit von Shengchen et al. konnte hingegen eine Reduktion der stationären Verweildauer und der Therapiedauer mit einem Antibiotikum um jeweils 1 Tag unter Verwendung eines Multiplex-PCR-Verfahrens als „point-of-care-testing“ (POCT) im Vergleich zu klassischen Routinetestungen gezeigt werden [28].

Von größerem Nutzen könnte der frühe Nachweis einer Virusinfektion bei Patienten mit umfangreichen Begleiterkrankungen, bei Vorliegen einer medikamentösen Immunsuppression oder immunsuppressiven Erkrankungen und der schweren CAP sein.

\section{Infektionen mit nicht- tuberkulösen Mykobakterien}

Nichttuberkulöse Mykobakterien (NTM) sind meist aus der Umwelt (Boden, Wasser, Oberflächen) erworbene Keime. Bei bestimmten Prädispositionen wie strukturellen Lungenerkrankungen oder Immunsuppression kann es zu einer Kolonisation bzw. zu einer Infektion mit resultierender Lungenerkrankung kommen. Daher bedarf es einer besonderen Aufmerksamkeit und gezielter Diagnostik bei diesen Patienten.

\section{》) In den letzten Jahren haben NTM-Isolate und -Erkrankungen zugenommen}

Mit Zunahme der immunsuppressiven und länger währenden tumorspezifischen Therapien, aber auch durch die geänderte Altersstruktur, wurde über die letzten Jahre ein Anstieg von NTM-Isolaten und NTM-Erkrankungen registriert [25].

In einer amerikanischen Studie wurde eine 5- bis 10-fach höhere NTMErkrankungsrate für Patienten mit anti-
TNFa-Therapie im Vergleich zu nichtbehandelten Patienten gezeigt. Die medikamentenspezifischen Inzidenzraten waren in aufsteigender Reihenfolge für Etanercept, Infliximab und Adalimumab angegeben [34]. Ein erhöhtes Risiko für NTM-Erkrankung ist auch bei der Behandlung mit anderen Immunsuppressiva wie z.B. Rituximab (anti-CD20-Antikörper), Abatacept (T-cell costimulator modulator), Tocilizumab (anti-IL-6-Antikörper) und Ustekinumab (anti-IL-12Antikörper) anzunehmen. Hierfür gibt es aber keine ausreichend validierten Daten, sodass weitere Studien notwendig sind [21].

Bei den Patienten mit vorbestehenden chronischen Lungenerkrankungen können sich die Symptome der Grunderkrankung mit den unspezifischen und variablen Symptomen einer pulmonalen NTM-Erkrankung überlappen.

Trotz verbesserter radiologischer und mikrobiologischer Diagnostik ist die Diagnosestellung einer klinisch relevanten NTM-Erkrankung und die Abgrenzung $\mathrm{zu}$ einer möglichen Besiedelung eine Herausforderung. Wichtig ist daher die korrekte Diagnosefindung unter Berücksichtigung der klinischen, radiologischen und mikrobiologischen Befunde anhand der IDSA/ATS-Leitlinie bzw. den Guidelines der British Thoracic Society (BTS) und der zu erwartenden neuen internationalen Leitlinie der verschiedenen Fachgesellschaften (ATS/IDSA, der European Respiratory Society [ERS] und European Society of Clinical Microbiology and Infectious Diseases [ESCMID]; $[9,12])$.

Die Behandlung einer Lungenerkrankung durch NTM ist medizinisch anspruchsvoll und langwierig, weist ein komplexes Nebenwirkungsprofil auf und birgt dadurch ein hohes Risiko eines Therapieabbruchs. Die Erfolgsrate der Therapie beträgt zwischen 40-60\% [31]. Das Therapieregime und der Beginn richten sich nach der Schwere der NTM-Erkrankung, der Erregerspezies und der Pathogenität des Erregers, dem zu erwartenden Krankheitsverlauf, den persönlichen Therapiezielen und den Begleiterkrankungen der häufig komorbiden Patienten. Zu beachten sind dabei die potenziellen Medikamenteninter- 
Tab. 1 Empfohlenes Therapieregime bei Patienten mit MAC-bedingten (M. avium complex) Lungenerkrankungen bei Erwachsenen. (Modifiziert nach [12],Brithish Thoracic Society guidelines for the management of non-tuberculous mycobacterial pulmonary disease [NTM-PD])

\section{Lungenerkrankung durch MAC}

Nichtschwere Lungenerkrankung durch MAC Sputum/Respirationsmaterial ohne Nachweis säurefester Stäbchen, radiologisch kein Nachweis von pulmonalen Kavernen oder schwerer Infektion, leichte bis mittlere Symptome, keine Zeichen einer systemischen Erkrankung

Schwere Lungenerkrankung durch MAC Sputum/Respirationsmaterial mit Nachweis säurefester Stäbchen, radiologischer Nachweis von Kavernen oder schwerer Infektion, ausgeprägte Symptome/Zeichen der Systemerkrankung

Clarithromycin-resistente Infektion durch $M A C$

\section{Antibiotikatherapie}

Rifampicin $600 \mathrm{mg} 3 \times$ pro Woche und Ethambutol $25 \mathrm{mg} / \mathrm{kg} 3 \times$ pro Woche und Azithromycin $500 \mathrm{mg} 3 \times$ pro Woche oder Clarithromycin $1 \mathrm{~g}$ in zwei Einzeldosen $3 \times$ pro Woche

Therapie sollte mindestens bis 12 Monate nach Kulturkonversion fortgeführt werden

Rifampicin $600 \mathrm{mg} / \mathrm{Tag}$ und Ethambutol $15 \mathrm{mg} / \mathrm{kg}$ pro Tag und Azithromycin $250 \mathrm{mg}$ pro Tag oder Clarithromycin $500 \mathrm{mg} 2 \times$ pro Tag; ggf. zusätzlich intravenöses Amikacin für bis zu 3 Monate oder Amikacin per inhalationem

Therapie sollte mindestens bis 12 Monate nach Kulturkonversion fortgeführt werden

Rifampicin $600 \mathrm{mg}$ pro Tag und Ethambutol $15 \mathrm{mg} / \mathrm{kg}$ pro Tag und Isoniazid $300 \mathrm{mg}$ (+Pyridoxine) pro Tag oder Moxifloxacin $400 \mathrm{mg}$ pro Tag; ggf. zusätzlich intravenöses Amikacin oder Amikacin per inhalationem Therapie sollte mindestens bis 12 Monate nach Kulturkonversion fortgeführt werden

aktionen zwischen der Vormedikation und der geplanten antimykobakteriellen Therapie. Resistenztestungen zur Optimierung und Individualisierung der Therapie bedürfen aktuell zumeist noch der kulturellen Erregeranzucht.

Anders als bei einer Infektion mit Mycobacterium tuberculosis ist auch eine engmaschige radiologische und klinische Kontrolle bei Patienten ohne Risikofaktoren unter Umständen zu rechtfertigen $[9,12]$. Exemplarisch ist in • Tab. 1 das empfohlene Therapieregime für $M y$ cobacterium avium complex (MAC), modifiziert nach den BTS-Guidelines, aufgeführt. Diese Empfehlung berücksichtigt die individuelle Krankheitssituation, aus der sich unterschiedliche Therapiemöglichkeiten ergeben.

\section{Bedeutung des Mikrobioms bei pulmonalen Infektionen}

Obwohl die Datenlagen bzgl. des Lungenmikrobioms noch immer sehr uneinheitlich und - stärker noch als bei anderen Lokalisationen des menschlichen Körpers - von methodischen Feinheiten abhängen, wurden zuletzt unterschiedliche Erkrankungen mit spezifischer $\mathrm{Zu}$ sammensetzung des Lungenmikrobioms assoziiert. Eine hervorragende Übersicht über den aktuellen Wissensstand zur Entwicklung des Lungenmikrobioms nach der Geburt, den Wechselwirkungen zwischen Immunsystem und Etablierung des Mikrobioms in den oberen und unteren Atemwegen sowie die zentralen Einflussgrößen für die Aufrechterhaltung eines "gesunden“" Mikrobioms findet sich in der Arbeit von Wypych et al. [35]. Dabei wird in den meisten Studien weiterhin das Mikrobiom als die Gemeinschaft bakterieller Isolate definiert, gleichwohl der Begriff im eigentlichen Sinn die Gemeinschaft aller mikrobiellen Erreger, also auch Viren und Pilze, einschließt.

\section{》) Bei Lungenerkrankungen und Infektionen nimmt die Diversität des Mikrobioms ab}

Bei respiratorischen Infektionen geht man davon aus, dass das Mikrobiom des Oropharynx und mutmaßlich auch des tieferen Respirationstrakts zunächst als Barriere vor einer Besiedlung und dann der Invasion potenziell pathogener Infektionserreger fungiert, darunter Bakterien aus der Gruppe der Firmicutes, Bacteroidetes und Proteobacteria, die sich in unterschiedlicher Zusammensetzung und Abundanz im oberen und unteren Respirationstrakt nachweisen lassen. Als ein generelles Merkmal unterschiedlichster Lungenerkrankungen (u. a. COPD, zystische Fibrose [CF]) und Infektionen (u.a. CAP, akute Exazerbation einer COPD [AECOPD]) findet sich eine Abnahme der Diversität des vorhandenen Mikrobioms und zumeist auch eine Zunahme von Proteobacteria, einer der größten Phyla gramnegativer Erreger [1, 7].

So konnten Tsang et al. zeigen, dass bei Haushaltsangehörigen die Übertragung von Influenza-A/B-Viren signifikant reduziert war, wenn die Personen im Nasen-Rachen-Abstrich mit Streptococcus sp. und Prevotella sp. kolonisiert waren [32]. Von Untersuchungen bei Kindern mit akuter RSV-Infektion weiß man, dass über eine Verknüpfung von Mikrobiom- mit Transkriptomdaten zur Wirtszellantwort der bei der Infektion involvierten Immunzellen Rückschlüsse auf den Schweregrad der Infektion gezogen werden können [30]. Aber auch für bislang gut charakterisierte Pathogene eröffnen sich durch die neuen „OMICs“-Verfahren (dt. -omiks) komplett neue Möglichkeiten, um Wirt-Pathogen- und Pathogen-Pathogen-Interaktionen zu untersuchen. Man findet bei CF-Patienten in gut einem Drittel der Patienten eine Koinfektion von S. aureus mit P. aerugino$s a$, was erhebliche Implikationen für das Voranschreiten der Erkrankung und die Auswahl geeigneter Antibiotika mit sich bringt [18]. Dabei eröffnen sich neue Strategien, die primär nicht nur die Eradikation eines potenziell pathogenen Erregers zum Ziel haben, sondern vielmehr auch den Schutz und den Erhalt protektiver Bakterien berücksichtigen.

Welches Potenzial in der Integration von Mikrobiomdaten zu bereits bekannten Prädiktoren einer Erkrankung steckt, zeigt nun erstmals eine Studie $\mathrm{zu}$ akuten Exazerbationen einer COPD (AECOPD). In einer Untersuchung an 102 Patienten in Vancouver/Kanada wurden bei Aufnahme mit der klinischen AECOPD-Symptomatik Sputumproben für 16S-Mikrobiom-Analysen abgenommen und die Sterblichkeit der Patienten über einen Zeitraum von 1 Jahr nachverfolgt [17]. Dabei zeigten sich signifikante Unterschiede in der Zusammensetzung 
der Mikrobiome von überlebenden und verstorbenen Patienten. So fand sich bei den später verstorbenen Patienten eine reduzierte Diversität des Mikrobioms mit einer Dominanz von Staphylococcus, wohingegen signifikant mehr Veillonella in den Sputumproben der Überlebenden nachweisbar waren [17].

\section{Fazit für die Praxis}

- Der Einsatz molekularer Testverfahren zur erweiterten Erregerdiagnostik ist im klinischen Alltag weiterhin auf Risikogruppen beschränkt oder wird meist nur im Rahmen saisonaler Influenzainfektionen eingesetzt.

- Aktuelle Studien zeigen uneinheitliche Ergebnisse hinsichtlich der Reduktion von Antibiotika und der Verringerung der Krankenhausliegedauer; belastbare Daten zu Morbidität und Mortalität in kontrollierten Studien liegen bislang nicht vor. Hingegen scheint ein Nutzen bei immunsupprimierten Patienten und bei V.a. auf seltene Infektionserreger oder im Rahmen von Ausbruchsgeschehen gegeben.

- Untersuchungen in klinischen Studien zum Lungenmikrobiom bei akuten Exazerbationen einer COPD zeigen erstmals eine direkte Assoziation mit Frequenz und Verlauf der Erkrankung.

- Die Integration der Erkenntnisse zum Mikrobiom könnte - zusammen mit neueren Befunden zu klinischen Biomarkern - bestehende Diagnosealgorithmen bei respiratorischen Infektionen optimieren, sofern sie in klinischen Studien einen direkten Nutzen zeigen.

\section{Korrespondenzadresse}

Prof. Dr. med. Jan Rupp

Klinik für Infektiologie und Mikrobiologie, Universitätsklinikum Schleswig-Holstein/ Campus Lübeck

Ratzeburger Allee 160, 23538 Lübeck, Deutschland

Jan.Rupp@uksh.de
Einhaltung ethischer Richtlinien

Interessenkonflikt. R. Hörster und J. Rupp geben an, dass kein Interessenkonflikt besteht.

Für diesen Beitrag wurden von den Autoren keine Studien an Menschen oder Tieren durchgeführt. Für die aufgeführten Studien gelten die jeweils dort angegebenen ethischen Richtlinien.

\section{Literatur}

1. Budden KF, Shukla SD, Rehman SF et al (2019) Functional effects of the microbiota in chronic respiratory disease. Lancet Respir Med 7:907-920

2. Dalhoff K, Abele-Horn M, Andreas S et al (2018) Epidemiology, diagnosis and treatment of adult patients with nosocomial pneumonia-update 2017—S3 guideline. Pneumologie 72(1):15-63

3. Das D, Le Floch H, Houhou N et al (2015) Viruses detected by systematic multiplex polymerase chain reaction in adults with suspected community-acquired pneumonia attending emergency departments in France. Clin Microbiol Infect 21(608):e1-8

4. Domenech de Cellès $M$, Arduin $H$, Lévy-Bruhl D et al (2019) Unraveling the seasonal epidemiology of pneumococcus. Proc Natl Acad Sci U S A 116:1802-1807

5. Ewig S, Höffken G, Kern WV et al (2016) Management of adult community-acquired pneumonia and prevention-update 2016. Pneumologie 70:151-200

6. Falsey AR, Hennessey PA, Formica MA et al (2005) Respiratory syncytial virus infection in elderly and high-riskadults. NEngl J Med 352:1749-1759

7. Faner R, Sibila O, Agustí A et al (2017) The microbiome in respiratory medicine: current challenges and future perspectives. Eur Respir J 1602086:49

8. González-Sanz R, Taravillo I, Reina J et al (2019) Enterovirus D68-associated respiratory and neurological illness in Spain, 2014-2018. Emerg Microbes Infect 8:1438-1444

9. Griffith $D E$, Aksamit T, Brown-Elliott BA et al (2007) An official ATS/IDSA statement: diagnosis, treatment, and prevention of nontuberculous mycobacterial diseases. Am J Respir Crit Care Med 175:367-416

10. Guery B, Poissy J, El Mansouf L et al (2013) Clinical features and viral diagnosis of two cases of infection with Middle East Respiratory Syndrome coronavirus: a report of nosocomial transmission. Lancet 381:2265-2272

11. Häussler B, Höer A (2018) Arzneimittel-Atlas 2018. Medizinisch Wissenschaftliche Verlagsgesellschaft, Berlin

12. Haworth CS, Banks J, Capstick T et al (2017) British Thoracic Society Guideline for the management of non-tuberculous mycobacterial pulmonary disease (NTM-PD). BMJ Open Respir Res 4:e242

13. Holter JC, MüllerF, Bjørang O et al (2015) Etiology of community-acquired pneumonia and diagnostic yields of microbiological methods: a 3-year prospective study in Norway. BMC Infect Dis 15:64

14. Johansson N, Kalin M, Hedlund J (2011) Clinical impact of combined viral and bacterial infection in patients with community-acquired pneumonia. Scand JInfect Dis 43:609

15. Kolditz M, Tesch F, Mocke L et al (2016) Burden and risk factors of ambulatory or hospitalized
CAP: a population based cohort study. Respir Med 121:32-38

16. LachantDJ, CroftDP, McGrane Minton Hetal (2017) Nasopharyngeal viral PCR in immunosuppressed patients and its association with virus detection in bronchoalveolar lavage by PCR. Respirology 22:1205-1211

17. Leitao Filho FS, Alotaibi NM, Ngan D et al (2019) Sputum microbiome is associated with 1-year mortality after chronic obstructive pulmonary disease hospitalizations. Am J Respir Crit Care Med 199:1205-1213

18. Limoli DH, Hoffman LR (2019) Help, hinder, hide and harm: what can we learn from the interactions between Pseudomonas aeruginosa and Staphylococcus aureus during respiratory infections? Thorax 74:684-692

19. Metlay JP, Waterer GW, Long AC et al (2019) Diagnosis and treatment of adults with communityacquired pneumonia. An official clinical practice guideline of the American Thoracic Society and Infectious Diseases society of America. Am J Respir Crit Care Med 200:e45-e67

20. Nationales Referenzzentrum für Surveillance von nosokomialen Infektionen Deutsche nationale Punkt-Prävalenzerhebung zu nosokomialen Infektionen und Antibiotika-Anwendung (2016) Abschlussbericht. https://www.nrz-hygiene.de/ fileadmin/nrz/download/pps2016/PPS_2016_ Abschlussbericht_20.07.2017.pdf

21. Novosad SA, Winthrop KL (2014) Beyond tumor necrosis factor inhibition: the expanding pipeline of biologic therapies for inflammatory diseases and their associated infectious sequelae. Clin Infect Dis 58:1587-1598

22. PickH, Daniel P, Rodrigo Cet al (2019) Pneumococcal serotype trends, surveillance and risk factors in UK adult pneumonia, 2013-18. Thorax. https:// doi.org/10.1136/thoraxjnl-2019-213725

23. Prendki V, Huttner B, Marti C et al (2019) Accuracy of comprehensive PCR analysis of nasopharyngeal and oropharyngeal swabs for CT-scan-confirmed pneumonia in elderly patients: a prospective cohort study. Clin Microbiol Infect 25:1114-1119

24. Quah J, Jiang B, Tan PC et al (2018) Impact of microbial aetiology on mortality in severe community-acquired pneumonia. BMC Infect Dis 18:451

25. Ringshausen FC, Apel RM, Bange FC et al (2013) Burden and trends of hospitalisations associated with pulmonary non-tuberculous mycobacterial infections in Germany, 2005-2011. BMC Infect Dis 13:231

26. Saarela E, Tapiainen T, Kauppila J et al (2019) Impact of multiplex respiratory virus testing on antimicrobial consumption in adults in acute care: a randomized clinical trial. Clin Microbiol Infect. https://doi.org/10.1016/j.cmi.2019.09.013

27. Self WH, Williams DJ, Zhu Y et al (2016) Respiratory viral detection in children and adults: comparing asymptomatic controls and patients with community-acquired pneumonia. J Infect Dis 213:584-591

28. Shengchen D, Gu X, Fan G et al (2019) Evaluation of a molecular point-of-care testing for viral and atypical pathogens on intravenous antibiotic duration in hospitalized adults with lower respiratory tract infection: a randomized clinical trial.Clin Microbiol Infect. https://doi.org/10.1016/ j.cmi.2019.06.012

29. Siljan WW, Holter JC, Michelsen AE et al (2019) Inflammatory biomarkers are associated with aetiology and predict outcomes in community- 


\section{Leitthema}

acquired pneumonia: results of a 5-year follow-up cohort study. ERJ Open Res 5:00014-2019

30. Sonawane AR, Tian L, Chu CY et al (2019)

Microbiome-transcriptome interactions related to severity of respiratory syncytial virus infection. Sci Rep 9:13824

31. Stout JE, Koh W-J, Yew WW (2016) Update on pulmonary disease due to non-tuberculous mycobacteria. Int JInfect Dis 45:123-134

32. Tsang TK, Lee KH, Foxman B et al (2019) Association between the respiratory microbiome and susceptibility to influenza virus infection. Clin Infect Dis. https://doi.org/10.1093/cid/ciz968

33. Wesemann T, Nüllmann H, Pflug MA et al (2015) Pneumonia severity, comorbidity and 1-year mortality in predominantly older adults with community-acquired pneumonia: a cohort study. BMCInfect Dis 15:2

34. Winthrop KL, Baxter R, Liu L et al (2013) Mycobacterial diseases and antitumour necrosis factor therapy in USA. Ann Rheum Dis 72:37-42

35. Wypych TP,Wickramasinghe LC, Marsland BJ(2019) The influence of the microbiome on respiratory health. Nat Immunol 20:1279-1290

36. Zhang Y, Sakthivel SK, Bramley A et al (2016) Serology enhances molecular diagnosis of respiratory virus infections other than influenza in children and adults hospitalized with community-acquired pneumonia.J Clin Microbiol 55:79-89

\section{SpringerMedizin.de}

\section{Lesen Sie Ihre Fachzeitschrift auch als ePaper!}

Als Abonnent können Sie Ihre Zeitschrift in verschiedenen Formaten lesen. Wählen Sie je nach Vorliebe und Situation aus, ob Sie die Zeitschrift als Print-Ausgabe, in Form von einzelnen Beiträgen auf springermedizin.de oder aber als komplette, elektronische ePaper-Ausgabe lesen möchten.

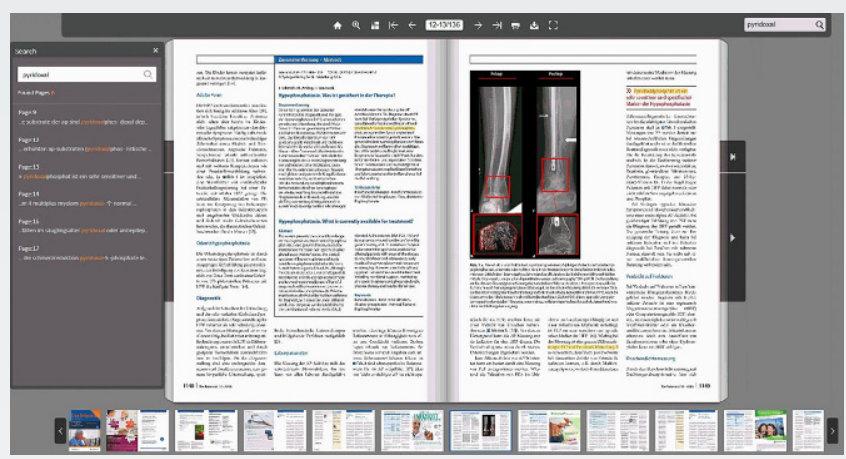

Die ePaper sind die identische Form der gedruckten Ausgaben. Sie sind nutzbar auf verschiedenen Endgeräten wie PC, Tablet oder Smartphone

Das sind die Vorteile des ePapers:

$>$ Das verlinkte Inhaltsverzeichnis führt Sie direkt zum gewünschten Beitrag.

$>$ Eine Suchfunktion ermöglicht das Auffinden von Schlagworten innerhalb der Zeitschrift.

> Jede Ausgabe kann als PDF heruntergeladen und damit auch offline gelesen werden bzw. auch gespeichert oder ausgedruckt werden.

> Als Abonnent haben Sie Zugang zu allen ePaper-Ausgaben ab 2016.

Sie finden die ePaper auf SpringerMedizin.de bei der jeweiligen Ausgabe Ihrer Fachzeitschrift. Klicken Sie auf den Button „Ausgabe als ePaper lesen“. 